\section{Evaluation of retinal nerve fiber layer and ganglion cell complex thickness after ocular blunt trauma}

G Cennamo ${ }^{1}$, R Forte ${ }^{1,2}, \mathrm{M}$ Reibaldi ${ }^{3}$, A Magli2, $^{2}$ $\mathrm{G}$ de Crecchio $^{1}$ and $\mathrm{G}$ Cennamo ${ }^{1}$
${ }^{1}$ Eye Department, University Federico II, Naples, Italy

\section{${ }^{2}$ Department of Ophthalmology, University of Salerno, Salerno, Italy \\ ${ }^{3}$ Department of Ophthalmology, University of Catania, Catania, Italy \\ Correspondence: R Forte, Department of Ophthalmology, University Federico II, Via Pansini 5, 80131 Naples, Italy. Tel: +393397662888; Fax: +39081425845 . \\ E-mail: raifor@hotmail.com}

Received: 28 March 2013 Accepted in revised form: 29 July 2013

Published online:

13 September 2013

\begin{abstract}
Purpose To evaluate circumpapillary retinal nerve fiber layer (cpRNFL) and ganglion cell complex (GCC) after ocular blunt trauma. Methods Best-corrected visual acuity (BCVA), cpRNFL and GCC were evaluated by RTVue-100 OCT in all consecutive patients with previous monocular blunt trauma seen between January 2012 and December 2012.

Results Twenty-two patients (11 females, 11 males, mean age $43.9 \pm 14.2$ years) were included in the study. Patients were seen after a mean of $8.42 \pm 13.3$ (range, 0.08-55.3) years from ocular blunt trauma. BCVA was normal in $\mathbf{1 1}$ cases and was less than 0.4 LogMAR in all cases. In 9/22 patients (40.9\%), cpRNFL and GCC were reduced, whereas in one case an isolated reduction of GCC with normal cpRNFL was present. In patients with reduction of cpRNFL and GCC, mean BCVA was $0.17 \pm 0.17$ LogMAR. In $6 / 9$ patients $(66.6 \%)$ with cpRNFL and GCC reduction, BCVA was $\leq 0.1$ LogMAR.

Conclusion cpRNFL and GCC reduction may be present after ocular blunt trauma and may be associated with preserved visual acuity. Eye (2013) 27, 1382-1387; doi:10.1038/eye.2013.203; published online 13 September 2013

Keywords: ocular blunt trauma; ganglion cell; traumatic optic neuropathy; RNFL; visual loss; perimetry

\section{Introduction}

Optical coherence tomography (OCT) allows to objectively evaluate structural damage in vivo and has been used to assess damage to the circumpapillary retinal nerve fiber layer
\end{abstract}

(cpRNFL) and to the ganglion cell complex (GCC). ${ }^{1-3}$ GCC includes the RNFL, retinal ganglion cell layer, and inner plexiform layer. ${ }^{2}$ Axonal loss after traumatic optic neuropathy (TON) has been shown through the use of OCT or scanning laser polarimetry. ${ }^{4-6}$ Recently, reduction of cpRNFL thickness and GCC has been found after unilateral TON. ${ }^{7}$

\section{Materials and methods}

All consecutive patients seen at the Eye Department of the University Federico II of Naples between January 2012 and December 2012 with a history positive for monocular blunt trauma were included in this cross-sectional study. The study protocol was approved by the institutional review board of Federico II University and adhered to the tenets of the Declaration of Helsinki. Written informed consent was obtained from each subject after explanation of the study. Inclusion criteria were a history of direct or indirect monocular ocular blunt trauma, according to previous charts reporting slit lamp examination and dilated fundus examination, without any intraocular disease and any blowout orbital or optic canal fracture, and intraocular pressure (IOP) $\leq 21 \mathrm{~mm} \mathrm{Hg}$. Exclusion criteria were presence of significant lens opacities or other media opacity as well any macular disease and actual or previous glaucoma therapies or suspect for low-tension glaucoma.

All patients underwent complete ophthalmic examination, including best-corrected visual acuity (BCVA) using standard Early Treatment of Diabetic Retinopathy Study (ETDRS, LogMAR), color fundus photography, stereophotographs of the optic disc, IOP 
measurement by applanation tonometry, and automatic perimetry (Humphrey Field Analyzer; Carl Zeiss Meditec, Dublin, CA, USA).

GCC and cpRNFL thickness were evaluated by RTVue-100 OCT (Optovue Inc., Fremont, CA, USA; software version 4.0.5.39). RTVue-100 OCT acquires 26000 axial scans (a-scans) per second and has a $5-\mu \mathrm{m}$ depth resolution (full-width half-maximum) in tissue. The optic nerve head map protocol was applied to evaluate the cpRNFL. This protocol generates a cpRNFL thickness map on the basis of measurements obtained along a circle $3.45 \mathrm{~mm}$ in diameter centered on the optic disc. The GCC protocol was used to determine the GCC and macular thickness. Only high-quality images, as defined by a signal strength index more than 30 , were accepted. The photographers rejected scans with motion artifacts (discontinuous jump). The GCC thickness was measured from the internal limiting membrane to the outer boundary of the inner plexiform layer. After examination a significance map with normative database comparison for GCC thickness was obtained using the device software. In both the study eyes and the contralateral eyes, the means of cpRNFL and GCC thickness were averaged over seven examinations, and contralateral eyes were used as controls. The ratio of the affected eye measurement to the contralateral eye measurement was evaluated at each session.
Statistical analysis was performed using the Statistical Package for Social Sciences (version 17.0, SPSS Inc., Chicago, IL, USA). Wilcoxon-signed rank test was used to compare study eyes and contralateral control eyes. Repeated measures ANOVA was used to compare intragroup differences. Results were considered significant if the $P$-value was $<0.05$.

\section{Results}

Twenty-two patients (11 females, 11 males, mean age $43.9 \pm 14.2$ years) were included in the study.

Characteristics of the 22 patients are resumed in Table 1. Patients were seen after a mean of $8.42 \pm 13.3$ (range, 0.08-55.3) years from ocular blunt trauma. In one case, trauma had occurred 1 month before our visit. In 10 cases, ocular blunt trauma occurred between 6 months and 1 year before our visit. Mean BCVA at examination was $0.07 \pm 0.13 \operatorname{LogMAR}$. BCVA was 0 LogMAR in 12 cases, $0.04 \log$ MAR in 3 cases, 0.1 LogMAR in 4 cases and 0.4 LogMAR in 3 cases. IOP was $\leq 21 \mathrm{~mm} \mathrm{Hg}$ in all eyes. Vertical cup/disc ratio was $<0.5$ in all eyes. Relative afferent pupillary defect, commotio retinae and optic atrophy at first examination were never observed.

In $9 / 22$ patients $(40.9 \%)$, both GCC and cpRNFL in the study eyes were reduced when compared with

Table 1 Demographics and clinical features of 22 consecutive patients that underwent monocular blunt trauma

\begin{tabular}{|c|c|c|c|c|c|c|c|c|}
\hline $\mathrm{N}$ & Gender & Eye & Cause of trauma & $\begin{array}{c}\text { Age at examination } \\
\text { (years) }\end{array}$ & $\begin{array}{c}\text { Time from trauma } \\
\text { (years) }\end{array}$ & $c p R N F L$ & GCC & $\begin{array}{c}\text { BCVA at examination } \\
\text { (ETDRS LogMAR) }\end{array}$ \\
\hline 1 & M & RE & Car accident & 27 & 0.5 & Normal & Normal & 0 \\
\hline 2 & M & $\mathrm{RE}$ & Car accident & 35 & 0.5 & Normal & Normal & 0 \\
\hline 3 & M & $\mathrm{RE}$ & Tennis ball & 57 & 0.7 & Normal & Normal & 0.04 \\
\hline 4 & $\mathrm{~F}$ & RE & Tennis ball & 33 & 0.7 & Normal & Normal & 0 \\
\hline 5 & M & RE & Party popper & 23 & 1 & Normal & Normal & 0 \\
\hline 6 & M & LE & Car accident & 25 & 0.5 & Normal & Normal & 0 \\
\hline 7 & M & $\mathrm{RE}$ & Blow by a fist & 35 & 0.5 & Normal & Normal & 0 \\
\hline 8 & M & LE & Car accident & 51 & 15.3 & Reduced & Reduced & 0.4 \\
\hline 9 & $\mathrm{~F}$ & RE & Car accident & 67 & 30.3 & Reduced & Reduced & 0.04 \\
\hline 10 & M & LE & Tennis ball & 54 & 10.3 & Reduced & Reduced & 0.04 \\
\hline 11 & $\mathrm{~F}$ & LE & Tennis ball & 30 & 0.8 & Reduced & Reduced & 0.4 \\
\hline 12 & F & LE & Car accident & 58 & 14.3 & Reduced & Reduced & 0.1 \\
\hline 13 & $\mathrm{~F}$ & $\mathrm{RE}$ & Car accident & 61 & 55.3 & Reduced & Reduced & 0.4 \\
\hline 14 & M & $\mathrm{RE}$ & Car accident & 51 & 12.3 & Reduced & Reduced & 0.1 \\
\hline 15 & $\mathrm{~F}$ & LE & Blow by a fist & 48 & 8.3 & Reduced & Reduced & 0 \\
\hline 16 & F & LE & Car accident & 59 & 1.1 & Normal & Normal & 0.1 \\
\hline 17 & F & $\mathrm{RE}$ & Car accident & 55 & 0.5 & Normal & Normal & 0 \\
\hline 18 & F & LE & Blow by a fist & 43 & 10.3 & Normal & Normal & 0 \\
\hline 19 & $\mathrm{~F}$ & $\mathrm{RE}$ & Tennis ball & 41 & 0.6 & Normal & Normal & 0 \\
\hline 20 & $\mathrm{~F}$ & LE & Blow by a fist & 56 & 20.4 & Reduced & Reduced & 0.1 \\
\hline 21 & M & $\mathrm{LE}$ & Car accident & 16 & 1.1 & Normal & Normal & 0 \\
\hline 22 & M & LE & Car accident & 41 & 0.08 & Normal & Reduced & 0 \\
\hline
\end{tabular}

Abbreviations: BCVA, best-corrected visual acuity; cpRNFL, circumpapillary retinal nerve fiber layer; F, female; GCC, ganglion cell complex; LE, left eye; $\mathrm{M}$, male; RE, right eye. 
contralateral eye $(91.4 \pm 17.0$ vs $99.8 \pm 7.1, P=0.01$ and $102.8 \pm 15.9$ vs $117.5 \pm 4.0, P<0.001$, respectively) (Figure 1 and Table 2). Only in one case with normal BCVA, an isolated reduction of GCC with normal cpRNFL thickness was present 1 month after trauma, and progressed during the following 2 months. Afterwards, 4 months after trauma, stabilization was observed (Figure 2). In this case, no perimetric abnormalities were detected during follow-up. In patients with reduction of cpRNFL and GCC, mean BCVA was $0.17 \pm 0.17$ LogMAR. In 6/9 patients $(66.6 \%)$ with cpRNFL and GCC reduction, BCVA was $\leq 0.1$ LogMAR. BCVA was 0.4 LogMAR in 3/9 cases $(33.3 \%)$ with reduction of both cpRNFL and GCC, after 15, 0.5, and 55 years from trauma (Figure 3). Of the 9 eyes with cpRNFL and GCC reduction, cpRNFL was reduced in the superotemporal quadrant in three cases, in the superonasal quadrant in one case, in the nasal quadrant in one case, in the temporal quadrant in two cases, in the inferotemporal quadrant in two cases. The interval from trauma was significantly longer in patients with cpRNFL and GCC reduction (18.6 \pm 15.9 vs $1.5 \pm 2.8$ years, $P<0.001)$. In $5 / 9$ eyes with cpRNFL and GCC reduction, diffuse non-glaucomatous perimetric defects were present, and in 3 of them BCVA was 0.4 LogMAR. In each case, distribution of cpRNFL reduction did not correspond exactly to distribution of visual field (VF) loss. In 4/9 eyes with cpRNFL and GCC reduction, no perimetric abnormality was found.

\section{Discussion}

In this retrospective observational study, we evaluated cpRNFL and GCC in patients who had undergone ocular blunt trauma. Of the 22 eyes with a history of ocular blunt trauma, 19 eyes (86.3\%) showed good visual acuity. cpRNFL and GCC thinning when compared with contralateral eye was present in 9/22 eyes (40.9\%). Of them, six eyes $(66.6 \%)$ showed preserved visual acuity, whereas moderate visual impairment was present in three eyes. Further to cpRNFL, the RTVue OCT device allows to evaluating GCC thickness, reflecting structural changes in the inner retina, including the retinal ganglion

Table 2 Axial length, ganglion cell complex (GCC) thickness and circumpapillary retinal nerve fiber layer (cpRNFL) thickness in the study eye and in contralateral control eyes

\begin{tabular}{lccl}
\hline & Study eye & Control & P \\
\hline Axial length $(\mathrm{mm})$ & $22.3 \pm 0.4$ & $22.3 \pm 0.5$ & 0.8 \\
GCC average $(\mu \mathrm{m})$ & $91.4 \pm 17.0$ & $99.8 \pm 7.1$ & 0.01 \\
GCC superior $(\mu \mathrm{m})$ & $88.4 \pm 17.7$ & $96.3 \pm 7.3$ & 0.01 \\
GCC inferior $(\mu \mathrm{m})$ & $94.5 \pm 18.5$ & $97.4 \pm 7.6$ & 0.1 \\
GCC S-I $(\mu \mathrm{m})$ & $-6.0 \pm 12.5$ & $-1.1 \pm 4.1$ & 0.001 \\
GCC FLV $(\%)$ & $3.5 \pm 5.6$ & $0.6 \pm 0.6$ & 0.001 \\
GCC GLV $(\%)$ & $10.7 \pm 10.6$ & $4.9 \pm 3.4$ & $<0.001$ \\
RNFL average thickness $(\mu \mathrm{m})$ & $102.9 \pm 15.9$ & $117.5 \pm 4.0$ & $<0.001$ \\
RNFL superior hemisphere $(\mu \mathrm{m})$ & $102.3 \pm 20.3$ & $120.5 \pm 6.5$ & $<0.001$ \\
RNFL inferior hemisphere $(\mu \mathrm{m})$ & $103.4 \pm 17.3$ & $114.5 \pm 3.0$ & $<0.001$ \\
\hline
\end{tabular}

Abbreviations: FLV, focal loss volume; GLV, global loss volume; S-I, superior-inferior difference.
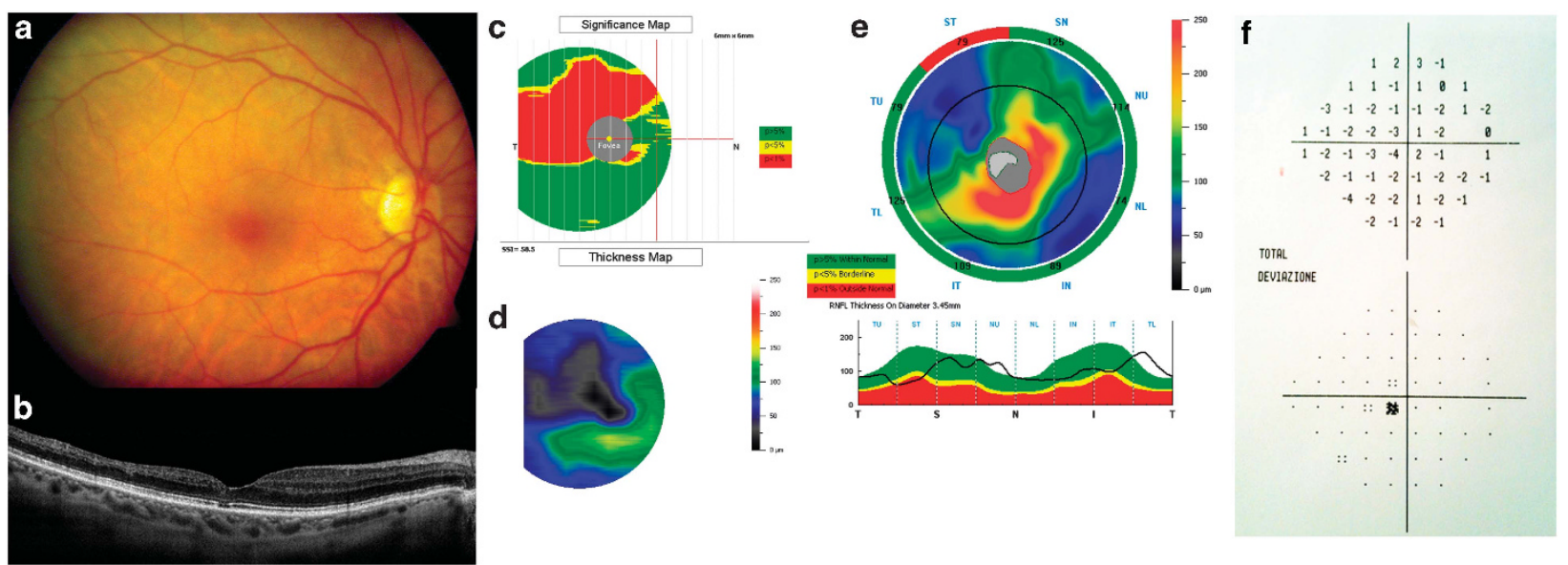

Figure 1 A 67-year-old female patient seen 30 years after ocular blunt trauma. Visual acuity is 0.04 LogMAR. (a) Color fundus image of the right eye. (b) At longitudinal OCT B-scan passing through the fovea, neuroretinal thinning temporal to the fovea is present. Reduction of GCC thickness is present (c) at significance map with normative database comparison and (d) at GCC thickness map. The Significance Map is color-coded where green represents values within the normal range ( $P$-value 5-95\%), yellow indicates Borderline results $(P$-value $<5 \%)$, and red represents an Outside Normal Limits ( $P$-value $<1 \%)$. (e) cpRNFL thickness is also reduced in the superotemporal sector. (f) Perimetry shows an isolated defect temporal to the fovea. No significant abnormalities were present in the contralateral eye. 
cells. Post-traumatic reduction of cpRNFL and GCC may follow traumatic glaucoma, TON and optic tract syndrome. Traumatic glaucoma may develop following damage to the aqueous drainage system or angle recession. In our study, both IOP and optic nerve head were normal in all patients, therefore traumatic glaucoma was excluded as a cause of cpRNFL and GCC reduction. Previous studies evaluated reductions in cpRNFL
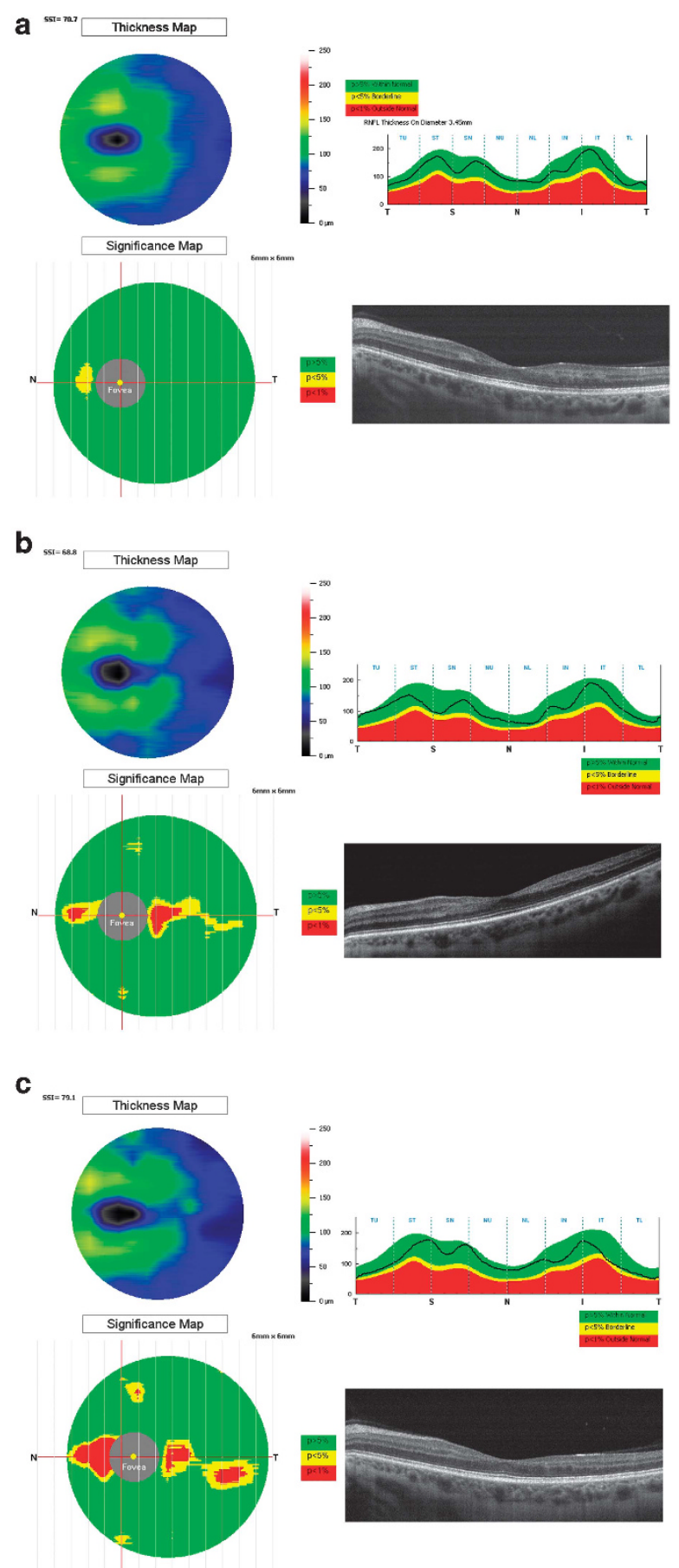

thickness after TON. TON determines severe visual impairment in association with heavy reduction of both cpRNFL and GCC. Three studies using scanning laser polarimetry reported that cPRNFL thickness did not decrease within 2 weeks after injury. ${ }^{4-6}$ Cunha et al ${ }^{8}$ showed that cpRNFL thickness started to decrease 2 weeks after injury. Kanamori et $a l^{7}$ showed progressive thinning of cpRNFL and GCC after TON using RTVue100 OCT in four patients with severe visual loss after a maximum follow-up of 9 months. cpRNFL and GCC thickness started to decrease at 2 weeks after trauma and plateaued at 20 weeks. Macular thickness also started to significantly decrease at 4 weeks after injury, which was in agreement with previous studies. ${ }^{8,9}$ The time course of the reduction in GCC thickness was similar to that observed for the cpRNFL. Therefore, the losses of retinal ganglion cells and related axons likely continue at similar rates as axonal injury. In all reported studies, severe visual impairment $(\leq 1.3 \log M A R)$ was present. In our series, visual impairment was not severe, although cpRNFL and GCC reduction was present. Only in one case, an isolated progressive GCC reduction with normal cpRNFL was present during first 3 months after trauma and stabilized thereafter. An ischemic insult to GCC may likely determine isolated GCC reduction with none or postponed cpRNFL reduction. Although in two cases seen 30 and 55 years after trauma, we cannot totally exclude a spontaneous or post-treatment improvement of TON as old data were not available, in the majority of cases $(20 / 22,91 \%)$ no data about TON were present in the clinical history and (in recent patients) at clinical examination. In our series, reduction of cpRNFL was localized in the superior and inferior quadrants, as well as in the nasal and temporal quadrants. Compression in the chiasmal region leads to significant reduction of the superior and inferior cpRNFL, ${ }^{10,11}$ and visual acuity may be preserved when temporal macular fibers are not damaged. Nasal and temporal cpRNFL reduction may follow optic tract syndrome, in which visual acuity may be preserved, and TON, which is characterized by

Figure 2 Progressive reduction of GCC thickness following ocular blunt trauma in a 41-year-old male patient. Visual acuity is 0 LogMAR throughout follow-up (counterclockwise from top left to top right: GCC thickness map, GCC significance map, longitudinal OCT B-scan passing through the fovea and cpRNFL thickness). (a) After 1 month from trauma, a focal reduction of GCC thickness nasal to the fovea is present. cpRNFL thickness is normal. No abnormalities are seen at longitudinal OCT B-scan passing through the fovea. (b) After 2 months from trauma, further thinning of GCC nasal and temporal to the fovea is present. Circumpapillary RNFL thickness and OCT B-scan are normal. (c) After 3 months from trauma, thinning of GCC is progressed and involves the superior quadrant. No abnormalities of circumpapillary RNFL and OCT B-scan are detectable. Stabilization was observed during the following months. 


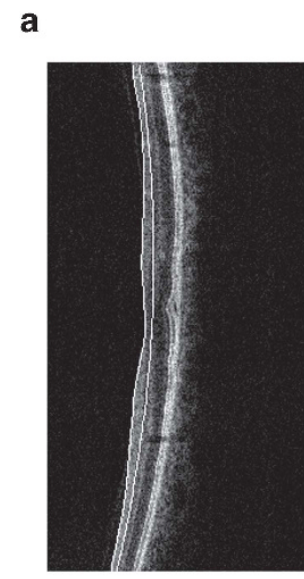

$$
\text { b }
$$
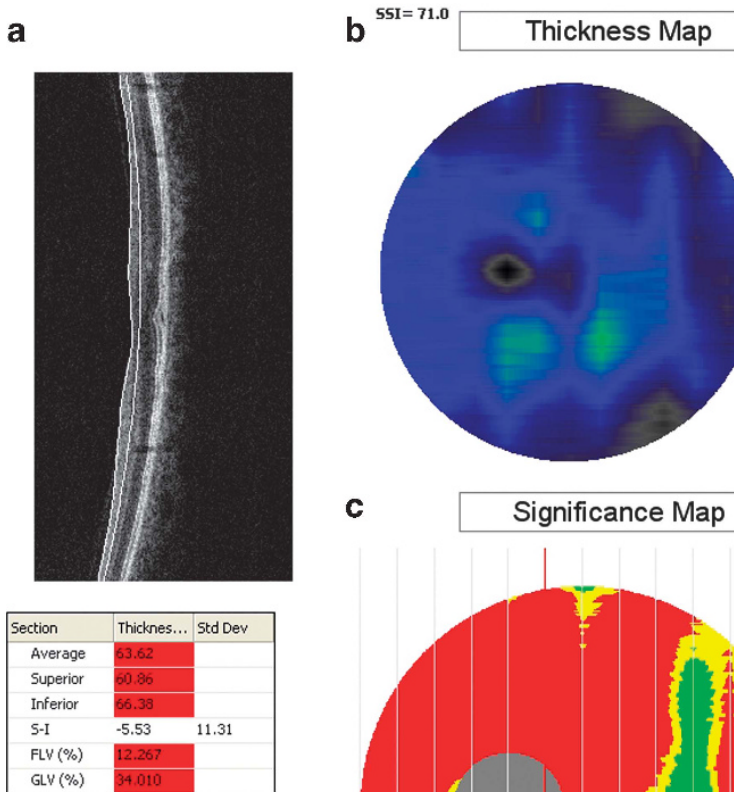

$b^{5 s 1=}$
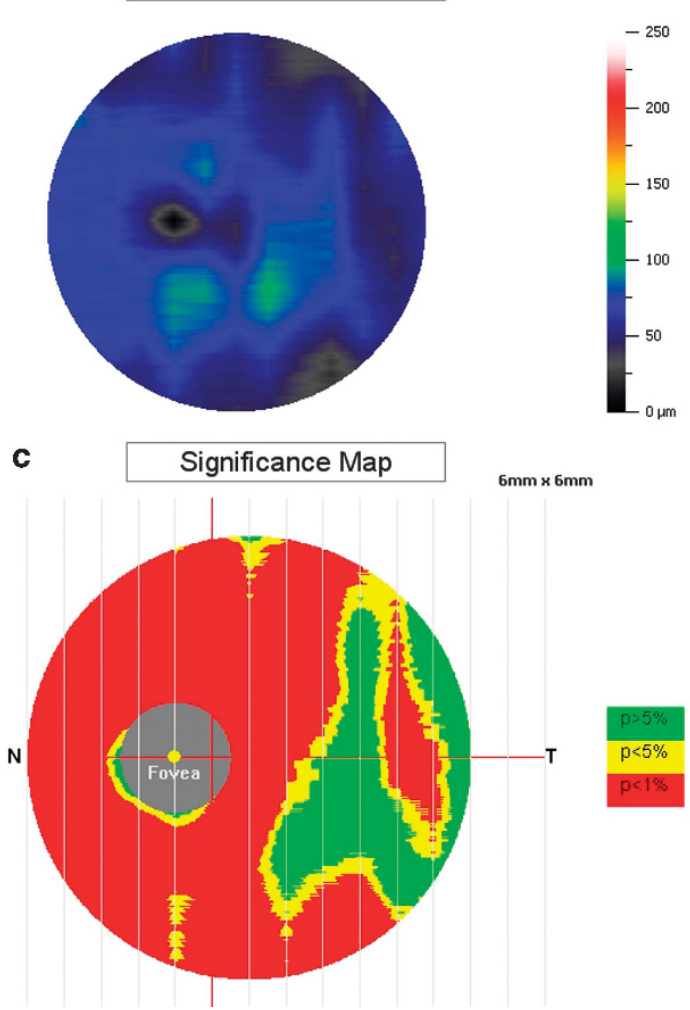

d
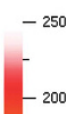

$-150$

$-100$

$-50$

$0 \mu \mathrm{m}$

e

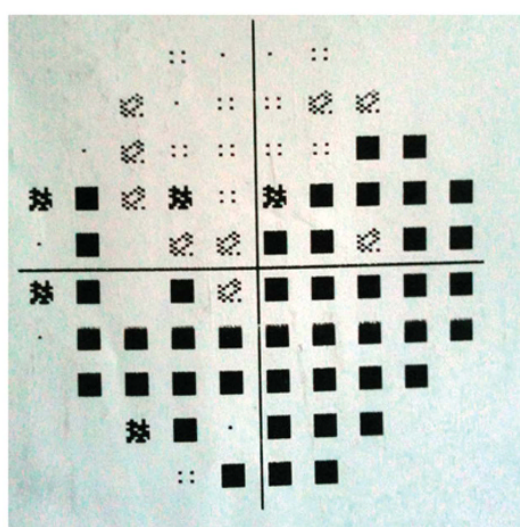

Figure 3 A 51-year-old male patient seen 15 years after ocular blunt trauma. Visual acuity is 0.4 LogMAR. (a) At longitudinal OCT B-scan passing through the fovea, neuroretinal thinning is not evident. Massive reduction of GCC at (b) significance map and (c) GCC thickness map, and (d) diffuse thinning of cpRNFL are present. (e) Reduction of sensibility and marked inferior VF loss are present at VF examination. The discrepancy between diffuse reduction of GCC and relative sparing of superior VF may be explained by the known minimum expected fiber loss before VF loss occurs. The contralateral eye was normal.

severely decreased visual acuity and relative afferent pupillary defect. ${ }^{7,12}$ According to our results, cpRNFL and GCC reduction in absence of severe visual loss could reflect a retrograde damage following chiasmic compression with sparing of temporal fibers or optic tract syndrome. Post-traumatic macular discoloration has been related to an outer retinal segment disruption rather than to the presence of an intraretinal edema, because there is no histological evidence for fluid accumulation in acute traumatic maculopathy. ${ }^{13,14}$ In a recent study by Saleh et al ${ }^{15}, 6$-month follow-up after trauma has shown no thinning of the cpRNFL, while an overall hyperreflectivity of the external retinal layers was present at SD-OCT examination. The blood supply from the nerve fiber layer to the external aspect of the inner nuclear layer is provided by the retinal vessels, the remainder of the retina is dependent on the choroidal circulation. Branches from the central retinal vessels dive deep into the retina forming two distinct capillary beds, one in the ganglion cell layer (superficial capillary plexus) and the other in the inner nuclear layer (deep capillary plexus). Blunt trauma determines a sudden increase of the IOP and consequent compression of the retinal vessels may happen, leading to focal ischemic damage to the superficial capillary plexus or both capillary beds and possible sufferance of the cpRNFL and GCC. According to our results, cpRNFL and GCC thinning with moderate or absent visual impairment could be related to a macular circulatory disturbance.

In 5/9 eyes with cpRNFL and GCC reduction, non-glaucomatous diffuse sensitivity reduction at automatic perimetry was present, and in 3 cases was associated with visual impairment. A disagreement between distribution of RNFL reduction and that of VF loss was present in each of the five cases. On the other hand, in 4/9 eyes with cpRNFL and GCC reduction and in one eye with GCC reduction and normal cpRNFL, no perimetric abnormality and good visual acuity were found. This likely suggests that fibers loss and VF defects do not show an exact correlation maybe due to the necessity for a minimum expected fiber loss before VF loss occurs, as already stated in previous studies. ${ }^{16}$ 
The interval from trauma was significantly longer in patients with cpRNFL and GCC reduction when compared with patients without abnormalities. However, the reductions in cpRNFL thickness and GCC after trauma are reported to plateaue at 20 weeks, ${ }^{7}$ and we could consider this being the maximum interval for damage to be excluded.

A limitation of this study is the retrospective design. Furthermore, in 10 cases, the ocular blunt trauma occurred only between 6 months and 1 year before our visit. However, the reduction in cpRNFL and GCC thickness has been showed to start 2 weeks after trauma and to plateau at 20 weeks. $^{7}$ Therefore, after 6 months from trauma, cpRNFL and GCC thickness can be assumed to have reached the maximum degree of reduction.

In conclusion, ocular blunt trauma may determine reduced cpRNFL and GCC thickness without visual impairment. Possible etiology of these cases may be a direct damage to ganglion cells during traumatic maculopathy or a retrograde damage following optic tract syndrome or chiasmal compression. A prospective evaluation is planned including pattern electroretinogram to detect cell dysfunction before apoptosis.

\section{Summary}

\section{What was known before}

- RNFL and ganglion cell complex have been evaluated in traumatic optic neuropathy with visual loss.

What this study adds

- Reduction of RNFL and ganglion cell complex may happen after ocular blunt trauma even in absence of visual loss.

\section{Conflict of interest}

The authors declare no conflict of interest.

\section{Acknowledgements}

The study was performed with informed consent and following all the guidelines for experimental investigations required by the Institutional Review Board or Ethics Committee of which all authors are affiliated.

\section{References}

1 Medeiros FA, Moura FC, Vessani RM, Susanna Jr R. Axonal loss after traumatic optic neuropathy documented by optical coherence tomography. Am J Ophthalmol 2003; 135: 406-408.

2 Tan O, Chopra V, Lu AT, Schuman JS, Ishikawa H, Wollstein G et al. Detection of macular ganglion cell loss in glaucoma by Fourier-domain optical coherence tomography. Ophthalmology 2009; 116: 2305-2314; e1-2.

3 Kim NR, Lee ES, Seong GJ, Kim JH, An HG, Kim CY. Structure-function relationship and diagnostic value of macular ganglion cell complex measurement using Fourier-domain OCT in glaucoma. Invest Ophthalmol Vis Sci 2010; 51: 4646-4651.

4 Medeiros FA, Susanna Jr R. Retinal nerve fiber layer loss after traumatic optic neuropathy detected by scanning laser polarimetry. Arch Ophthalmol 2001; 119: 920-921.

5 Meier FM, Bernasconi P, Stürmer J, Caubergh MJ, Landau K. Axonal loss from acute optic neuropathy documented by scanning laser polarimetry. Br J Ophthalmol 2002; 86: 285-287.

6 Miyahara T, Kurimoto Y, Kurokawa T, Kuroda T, Yoshimura N. Alterations in retinal nerve fiber layer thickness following indirect traumatic optic neuropathy detected by nerve fiber analyzer GDx-N. Am J Ophthalmol 2003; 136: 361-364.

7 Kanamori A, Nakamura M, Yamada Y, Negi A. Longitudinal study of retinal nerve fiber layer thickness and ganglion cell complex in traumatic optic neuropathy. Arch Ophthalmol 2012; 130: 1067-1069.

8 Cunha LP, Costa-Cunha LV, Malta RF, Monteiro ML. Comparison between retinal nerve fiber layer and macular thickness measured with OCT detecting progressive axonal loss following traumatic optic neuropathy. Arq Bras Oftalmol 2009; 72: 622-625.

9 Vessani RM, Cunha LP, Monteiro ML. Progressive macular thinning after indirect traumatic optic neuropathy documented by optical coherence tomography. $\mathrm{Br}$ J Ophthalmol 2007; 91: 697-698.

10 Kanamori A, Nakamura M, Matsui N et al. Optical coherence tomography detects characteristic retinal nerve fiber layer thickness corresponding to band atrophy of the optic discs. Ophthalmology 2004; 111: 2278-2283.

11 Monteiro MLR, Leal BC, Rosa AAM et al. Optical coherence tomography analysis of axonal loss in band atrophy of the optic nerve. Br J Ophthalmol 2004; 88: 896-899.

12 Tatsumi Y, Kanamori A, Kusuhara A, Nakanishi Y, Kusuhara S, Nakamura M. Retinal nerve fiber layer thickness in optic tract syndrome. Jpn J Ophthalmol 2005; 49: 294-296.

13 Gregor Z, Ryan SJ. Blood-retinal barrier after blunt trauma to the eye. Graefes Arch Clin Exp Ophthalmol 1982; 219: 205-208.

14 Hart JC, Blight R. Commotio retinae. Arch Ophthalmol 1979; 97: 1738.

15 Saleh M, Letsch J, Bourcier T, Munsch C, Speeg-Schatz C, Gaucher D. Long-term outcomes of acute traumatic maculopathy. Retina 2011; 31: 2037-2043.

16 Ferreras A, Pablo LE, Garway-Heath DF, Fogagnolo P, García-Feijoo J. Mapping standard automated perimetry to the peripapillary retinal nerve fiber layer in glaucoma. Invest Ophthalmol Vis Sci 2008; 49: 3018-3025. 